\title{
Wake characterization of an undulating membrane tidal energy converter
}

\author{
Träsch Martin 1, 2, 3, * , Déporte Astrid 1, Delacroix Sylvain 1, Germain Gregory 2, Gaurier Benoit 2, \\ Drevet Jean-Baptiste ${ }^{3}$
}

1 EEL ENERGY, 60, rue de Folkestone 62200 Boulogne sur Mer, France

2 IFREMER, Marine Structure Laboratory, 150 Quai Gambetta 62200 Boulogne sur Mer, France

${ }^{3}$ Ademe, 20 avenue du Gresille, Angers 49004, France

* Corresponding author : Martin Träsch, email address : $\underline{\text { mtrasch@eel-energy.fr }}$

\begin{abstract}
:
Most of the research done on tidal energy focuses on marine current turbines. Therefore, tidal turbine's wake is well-documented. The tidal energy converter studied here is based on the fluid-structure interactions that occur between a flexible membrane and an axial flow, resulting in an undulating motion that can be used to harvest energy. This device's performance had been studied but it is the first time its wake is experimentally characterized from two-dimensional Particle Image Velocimetry (PIV) measurements. PIV is synchronized with a motion tracking system that gives information on trajectory and power conversion. Wake measurement gives access to velocity deficit, turbulence intensity and vorticity. Three configurations are tested in order to identify the influence of the main adjustment parameters. Pre-stress increases the membrane vertical speed, leading to a more important vertical expansion of the wake. The power extraction slows down the membrane's motion, thus limiting the wake's length and intensity. The flume tank measurements suggest that the best location for a downstream device in a tidal farm would be in the same horizontal position, at 5 membrane's length. In the open sea, the membrane interaction effects should not predominant.
\end{abstract}

\section{Highlights}

- Undulating membrane first wake characterization from PIV measurements, $>$ Phase-averaged velocity deficit and vorticity are measured and presented, Vorticity dissipation rate result can be used to calibrate numerical model.

Keywords : Marine Renewable Energy, Tidal Energy, Undulation, Power Take-Off, Wake characteristics, Flume tank 


\section{Introduction}

Wake characterization takes an important part in tidal energy converters development. It is a requirement to design tidal energy converter arrays as it defines the optimal spacing between several devices [3]. It is also used in environmental impact studies to assess potential modification of local currents and sediment transport [19].

While horizontal and vertical axis tidal turbines' wakes have been already studied $[4,15,17,25]$ and their interaction effects well-known [16, 2], no measurement has been done on an undulating tidal energy converter. Indeed, the tidal energy converter studied here is based on the fluid-structure interactions that occur between a flexible membrane and an axial flow, resulting in an undulating motion that can be used to harvest energy. This device's performance has been studied [22] but its wake had never been quantified experimentally.

Flow patterns behind other undulating bodies have been studied and measured [14]. Their motion might be similar but the flow patterns they generate differ significantly as it comes from a passive flag or an active swimming fish. Their patterns can be formed of vortex ribbon, vortex streets and flow jets, or a combination of them (Fig. 1). Other studies focus on the role of the wake in flags interactions [24]. Most of them underline a coupling phenomenon, with in-phase or out-of-phase oscillations $[1,27]$. This confirms the importance of studying the wake of undulating membrane tidal energy converters in order to prepare a tidal farm interaction study.

Numerical simulation have also been undertaken to study the wake of cantilevered flexible plates in axial flow in order to develop wind converters $[10,18]$. The study presented here differs both by its approach: experimental, in water flume tank; and by its model: flexible plate is here bended by lateral cables and damped by hydraulic dampers.

A previous study on the Power Take-Off (PTO) modelled by hydraulic dampers fixed along the membrane revealed that they act on the undulating mode and frequency of the system [22]. The membrane is also pre-stressed by cables linking both extremities that keep the membrane bended (Fig. 2 ). The cables length influences the stability of the system, its undulations amplitude and the power conversion [23]. The wake of three experimental configurations are then studied here : a "free" configuration, with no PTO and a small pre-strain; an "intermediate" configuration, with PTO and small pre-strain; and an "efficient" configuration, with PTO and a greater prestrain. 
(A) Flag wake

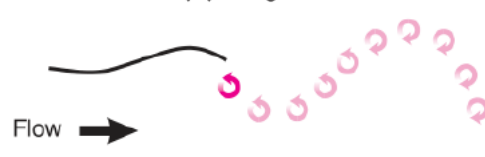

(B) Thrust wake

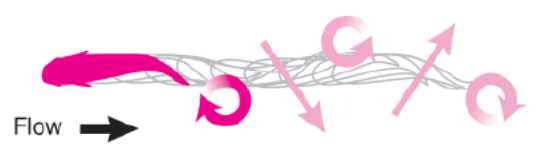

Figure 1: Vorticies (circles) and flow jets (arrows) behind (a) a passive flag and (b) a swimming fish in water flow [14].

In this paper, experimental model and data process are first described. The wake of three different configurations are studied from PIV measurements. The mean and instantaneous velocity, turbulence intensity and vorticity fields are presented and analysed.

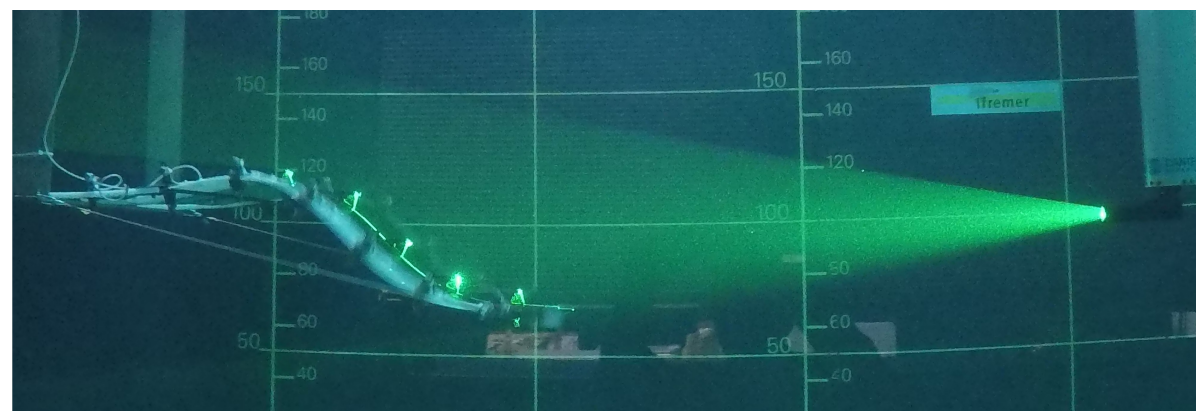

Figure 2: Picture of the experimental model during PIV measurements.

\section{Experimental set-up}

Experiments have been carried out in the Ifremer flume tank of Boulognesur-Mer (Fig. 3). Its working section is $18 \mathrm{~m}$ long, $4 \mathrm{~m}$ wide and $2 \mathrm{~m}$ deep. A window enables to film the membrane motion from the side in order to record its trajectory. Flow velocity is adjustable up to $2.2 \mathrm{~m} / \mathrm{s}$. Here, trials have been made at $u_{\infty}=1 \mathrm{~m} / \mathrm{s}$ with a mean upstream turbulence intensity rate of $I_{\infty}=1.5 \%$ (Eq. 1). The turbulence intensity characterizes the level of fluctuations in the flow as a percentage, it is calculated here on two components. $\sigma$ designates the standard deviation. 


$$
I_{2 D}=100 \sqrt{\frac{1}{2} \frac{\sigma\left(u^{\prime}\right)^{2}+\sigma\left(v^{\prime}\right)^{2}}{\bar{u}^{2}+\bar{v}^{2}}}
$$

The fluctuating velocity $\mathbf{u}^{\prime}$ is defined as the difference between the local instantaneous current speed and the mean flow speed (Eq. 2), with $u(t)$ the horizontal velocity component and $v(t)$ the vertical one.

$$
\mathbf{u}^{\prime}=\left(u^{\prime}(t), v^{\prime}(t)\right)=(u(t)-\bar{u}, v(t)-\bar{v})
$$

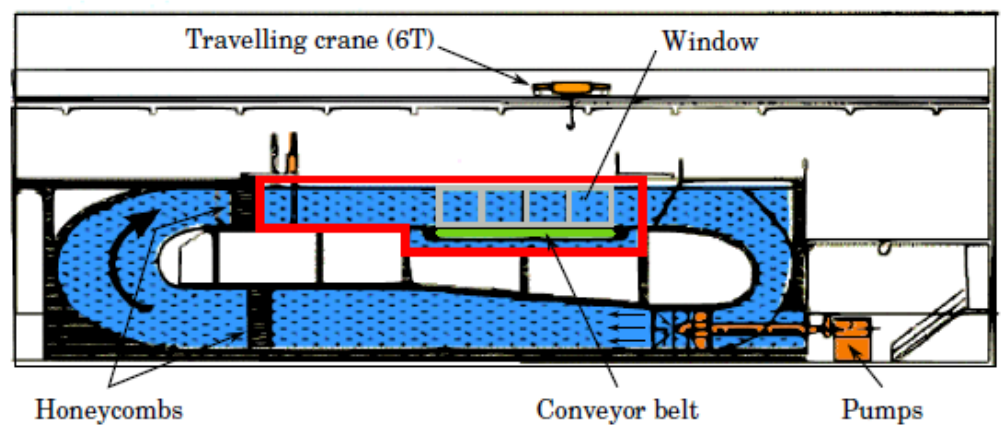

Figure 3: Cutaway drawing of Ifremer's flume tank of Boulogne-sur-Mer.

The prototype used in the experiments is at $1 / 20^{\text {th }}$ scale. It is made of a POM-C polyacetal membrane of dimensions $L \times b \times h=0.8 \mathrm{~m} \times 0.8$ $\mathrm{m} \times 0.003 \mathrm{~m}$, density $\rho=1600 \mathrm{~kg} / \mathrm{m}^{3}$ and Young modulus $E=4.2 \mathrm{GPa}$ [22]. Blockage ratio is then about $B=L A /\left(S_{\text {canal }}\right) \approx 0.04$, with $A$ the undulation amplitude.

This membrane is lengthened by $0.15 L$-long rigid flaps at upstream and downstream extremities. In order to increase the transverse stiffness and to ensure a two-dimensional motion, six $0.025 \mathrm{~L}$-wide carbon-epoxy bars have been added in the transverse direction. They are separated by $0.2 L$ on the membrane and transmit forces to the converters through pivot fixations of height $h_{\text {fix }}=0.044 L$ (Fig. 4).

To keep the structure in the middle of the water column, the membrane is clamped to a rigid vertical frame with three horizontal POM-C bars of dimensions $0.250 \mathrm{~m} \times 0.046 \mathrm{~m} \times 0.010 \mathrm{~m}$. The structure is also stressed by cables linking both extremities that keep the membrane bended (Fig. 4). The cables are shorter than the resting distance between their linking points. Their length is described through the cable withdraw length, $d$, as defined by Equation 3. Two withdraw length values will be considered in this paper: $7 \%$ and $12.5 \%$. 


$$
d=\frac{L+L_{a r m}-L_{\text {cable }}}{L}
$$
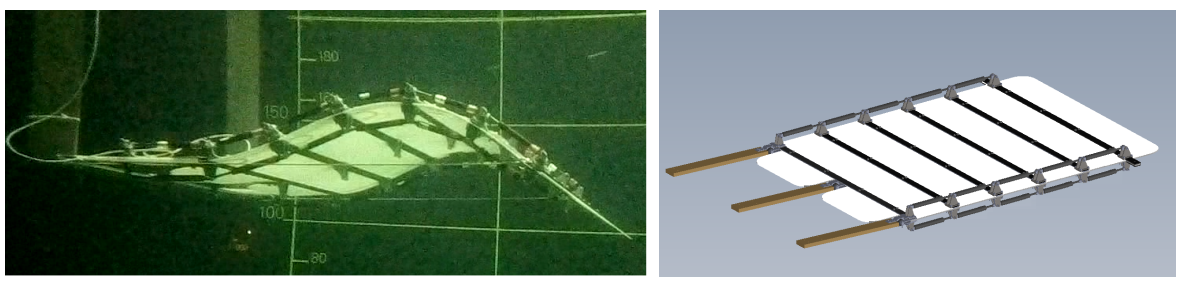

Figure 4: (a) Picture of $1 / 20^{t h}$ scale prototype during experiments in Ifremer flume tank of Boulogne-sur-Mer. (b) CAD 3D view of tested prototype. Membrane is in white, transverse bars in light grey, damper's fixation in dark grey and dampers in black.

At this scale, it is easier to dissipate energy than to convert it into electricity. Therefore, for physical and mechanical constraints, electromechanical converters are modeled by hydraulic dampers, as it has been experimented for oscillating body devices in wave converter models [5]. Four lines of 6 dampers are installed on the sides of the membrane, two above and two bellow (Fig. 4). The 24 dampers used in this study are the HB15/75/S/S/B from Slamproof Ltd. They are characterized before and after experiments on a displacement table instrumented with a 2000DaN S-type load cell. This is in order to verify the dampers' adjustment and to estimates their damping force and power dissipation more precisely. Imposed trajectory on the displacement table is close to operating ones: it is a sine with $0.3 \mathrm{~Hz}$ frequency and $30 \mathrm{~mm}$ amplitude. Force $F$ and velocity $V$ are measured on the displacement table and the signals are filtered by a $3 \mathrm{~Hz}$ low-pass filter. The damping rate $C(\mathrm{~kg} / \mathrm{s})$ is defined as the mean value of $F / V$, for all values of $V \leq 10 \mathrm{~mm} / \mathrm{s}$. It is then normalized by the ratio between the flow speed and the membrane's stiffness (Eq. 4), $I_{z}$ being the second moment of area in the transverse direction. Non-dimensional damping is here equal to $C^{*}=300$.

$$
C^{*}=C \frac{L^{2} u_{\infty}}{E I_{z}}
$$

The wake of three experimental configurations are studied : a "free" configuration, with no PTO and a small pre-strain, an "intermediate" configuration, with PTO and small pre-strain, and an "efficient" configuration, with PTO and a greater pre-strain. These configurations have been selected because they are close to the state-of-the art efficient configuration that are used in the other studies on this device. Moreover, they have been selected 
to give an insight of the effect of damping and pre-strain on the wake dynamics, since these two parameters are the main adjustment parameters of the device. Furthermore, these three configurations have three different levels of extracted energy, the "free" configuration being closer to systems whose wake are studied in the existing literature. A summary of tested configurations is presented in Table 1. The undamped configuration is lighter because of the removal of dampers' weight.

\begin{tabular}{|c|r|r|r|r|}
\hline Configuration & Mass & Dampers & Damping, $C^{*}$ & Withdraw length, $d$ \\
\hline 1 & $8.60 \mathrm{~kg}$ & none & 0 & $7 \%$ \\
2 & $10.1 \mathrm{~kg}$ & 24 & $24 \times 300$ & $7 \%$ \\
3 & $10.1 \mathrm{~kg}$ & 24 & $24 \times 300$ & $12.5 \%$ \\
\hline
\end{tabular}

Table 1: Summary of tested configurations.

Total dissipated power $P$ is estimated by Equation 5, as the sum of the products between activation velocity $V(t)$ and corresponding damping force $F(t)$ for $M$ dampers. Overbar indicates averaged value calculated over 10 periods

$$
P=\sum_{i=1}^{M} \overline{V_{i}(t) F_{i}(t)} \approx \sum_{i=1}^{M} \overline{C_{i} V_{i}^{2}(t)}
$$

Seven LEDs are located on the extremities of the dampers, and are used as targets for motion tracking. A Qualysis Oqus 310 camera (1024 px × $1296 \mathrm{px}$ ) is placed at $0.8 \mathrm{~m}$ in front of the side window to film the trials at a frame rate of $f_{e}=100 \mathrm{~Hz}$. Trajectories are obtained through the Automatic Identification of Markers (AIM) model of the Qualisys Track Manager software. It identifies trajectories by machine learning from angles and distances between markers.

Figure 5 gives the results of motion tracking. It shows a strong impact of cables length and damping on the motion amplitude. Moreover, it shows that the mean position is impacted by gravity. Results of non-dimensional frequency are respectively $f^{*}=f . L / u_{\infty}=0.55$ and 0.27 for undamped and damped configurations (Table 1). Power is estimated following the method described in [22], then normalized by their higher average value. Results are: $C p_{1}=0.0 ; C p_{2}=0.6 ; C p_{3}=1.0$. 


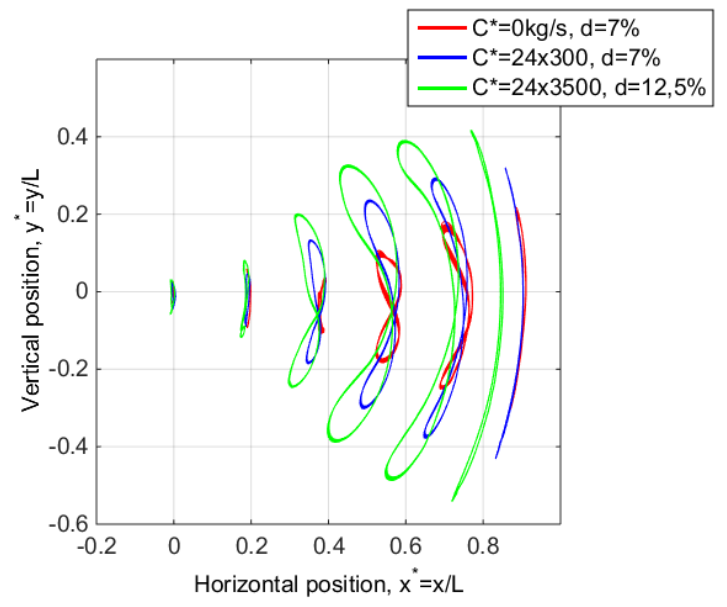

Figure 5: Trajectory of points in the membrane separated by $0.2 \mathrm{~L}$ for the three tested configurations : $C^{*}=0, d=7 \%$ (red), $C^{*}=24 \times 300, d=7 \%$ (blue) and $C^{*}=24 \times 300$, $d=12 \%$ (green),

In order to characterize the flow in the membrane's wake, measurements have been carried out by means of a 2D PIV system on a vertical plane along $x$ and $y$ axes and located at the middle of the membrane in the spanwise direction (Fig. 6). For these measurements, the tank has been seeded by silver-coated glass particles of diameter $10 \mu \mathrm{m}$. Illumination has been provided by a standard, frequency-doubled, double-cavity Nd:YAG GeminiLike laser with up to $200 \mathrm{~mJ}$ per pulse using an excitation wavelength of $532 \mathrm{~nm}$. The laser sheet is emitted in the water by means of an optical system composed of cylindrical lenses mounted on a vertical laser-guiding arm in order to generate a light sheet on vertical plane. PIV images pairs are recorded at $15 \mathrm{~Hz}$, with a low interval time of $\Delta t=1500 \mu$ s between the two frames of the pair [13]. The camera (Hi-sense CCD FlowSens EO-2M camera of $1400 \mathrm{px} \times 900 \mathrm{px})$ is located perpendicularly to the laser sheet. The PIV system is synchronized with the motion tracking system through a trigger device. The distance between the camera and the laser sheet is $2.2 \mathrm{~m}$, so that the PIV planes are located in the middle of the membrane in the transverse direction. Their dimensions are $970 \mathrm{~mm} \times 616 \mathrm{~mm}$. The uncertainty of the flow speed measured by PIV is estimated to be of $2.6 \%$ $[9]$. 


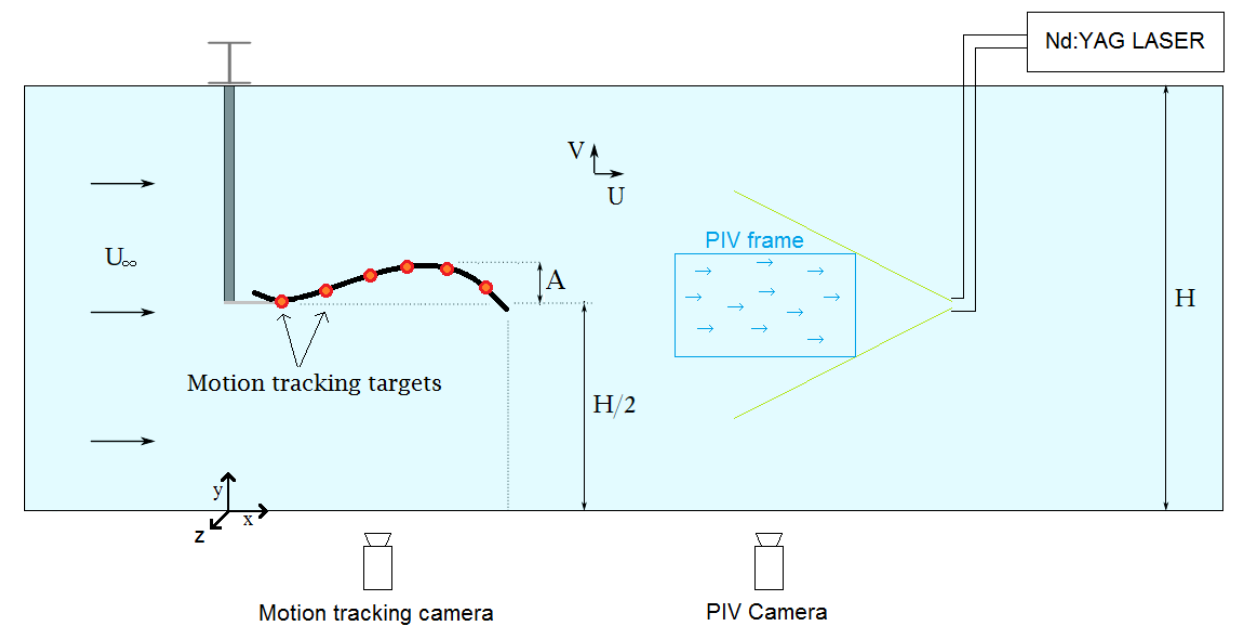

Figure 6: Drawing of the experiment instrumentation.

DynamicStudio software from Dantec Dynamics has been used for PIV image processing. Instantaneous velocity vector fields have been obtained using a single-pass cross-correlation algorithm with an interrogation window size of $32 \mathrm{px} \times 32 \mathrm{px}$, with $50 \%$ overlap. Streamwise ( $x$-direction) and vertical ( $y$-direction) velocity components are then available on a $2 \mathrm{D}$ regular mesh grid of $(86 \times 55)$ points with an element length of $11.4 \mathrm{~mm}$ in each direction.

Universal Outlier Detection (UOD) algorithm is used to locate and replace abnormal vectors [26]. It detects those being different by a factor 2 of the mean value of the $5 \times 5$ neighbor vectors. Abnormal vectors are then replaced by their neighbours' median value. Figure 7 summarizes the post-process stages on Dynamic Studio software.

The planes are registered one after the other so the post-process needs to put them back in space and time in order to generates global wake maps. Twenty-three planes have been registered during $300 \mathrm{~s}$ for each configuration, which enables to assemble planes on an area of $2.25 \times 10 L^{2}$. However, on the sides of the planes, particles are not well lightened by the laser and there are some abnormal vectors. $3 \mathrm{px}$ bands are then cut from analysis to remove them. Figure 8 presents the PIV planes layout with the tidal converter's trajectory superimposed. 

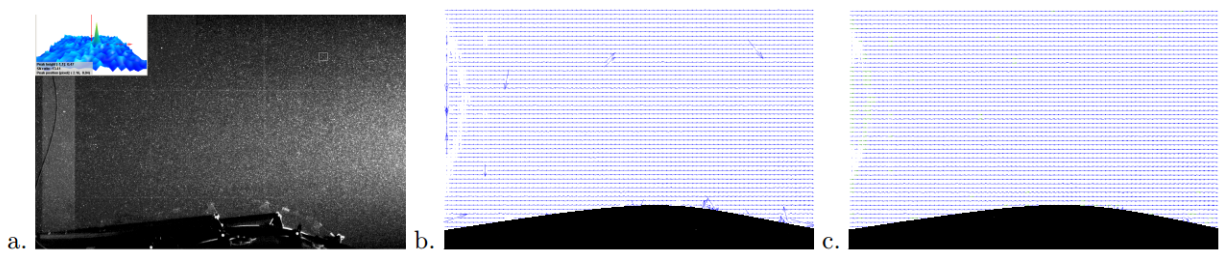

Figure 7: Post-processing stages on Dynamic Studio. (a) Raw image. (b) After crosscorrelation algorithm. (c) After UOD algorithm.

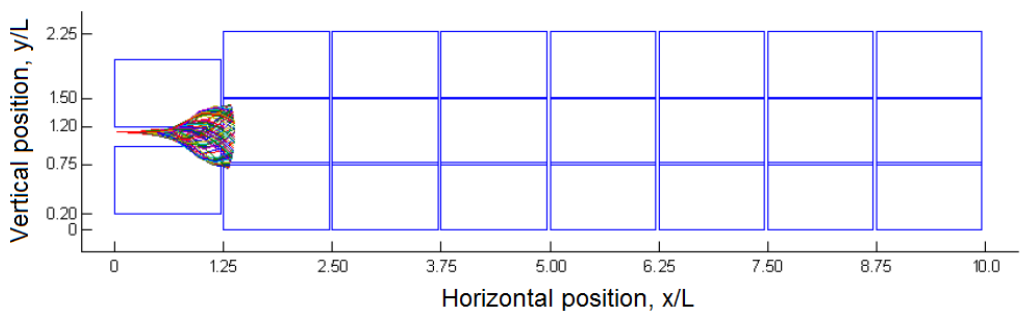

Figure 8: PIV planes location.

In order to synchronize every plane, motion tracking system is used to get the time step when the last target passes from below to above $y=1.15 \mathrm{~L}$, which is the membrane's fixation vertical position. As undulation frequency happens to vary, it is calculated in every plane and used to synchronize every period in order to calculate phase-averaged values. The uncertainty on the time a period starts is of the order of the sampling frequency inverse, $1 / f_{e}$ $=0.067 \mathrm{~s}$. Phase averages are then calculated with an uncertainty of the order of a hundredth of the undulation period.

The vorticity is defined as the curl of the velocity vector field. It describes the local spinning motion of the flow and is calculated by Equation 6 [8]. For a better readability, a spatial window average is applied on vorticity maps with a 5 pixels window.

$$
\omega=\frac{\rho}{2}\left(\frac{\partial u}{\partial y}-\frac{\partial v}{\partial x}\right)
$$




\section{Wake characterisation}

The results presented in this paper are the first wake measurements of undulating membrane tidal energy converter. They aim to give a first insight on the wake structure and characteristics of such devices, that have until now only been studied at small scale. Mean wake maps are first studied, then the velocity fluctuations. The wake structure is adressed through intensity turbulence and vorticy calculations. The next section presents comparison with axial turbine tidal converter and the wake impact the hydrokinetic power available downstream.

\subsection{Mean wake structure}

Mean axial velocity maps in the membrane's wake for the three tested configurations is presented in Figure 9. The mean axial velocity deficit is weak: $\max \left(\Delta u^{*}\right)=\max \left(1-\overline{u^{*}}\right) \approx 0.1$. It is also limited in length as it is no more significant after $x=5 L$ downstream, especially in $y^{*} \approx 0$. The wake of the third configuration $(d=12.5 \%)$ spreads faster vertically, and is slightly more important than the wake of the second one $(d=7 \%)$. The higher vertical expansion is caused by the highest membrane undulation amplitude generated by the the pre-stress cables being shorter. The higher velocity deficit is due to the additional energy extraction.

However, the wake of the first one is also more pronounced and persists further. It is due to the faster membrane motion arising from lack of damping. Indeed, its undulation frequency is twice the frequency of the damped cases. Moreover, the deformation of the undamped membrane is more important as it is not limited by the dampers' stroke.

Mean vertical velocity maps in the wake are presented Figure 10. It enables to spot two areas where mean vertical speed is impacted : it is positive in the upper area and negative in the lower area. Bottom area (negative vertical velocity) is wider and faster than its top counterpart. This is due to the gravity effect that drives the membrane to be faster when it goes down and slower when it rises (Fig. 15). Moreover, interaction with bottom floor and with free surface might accentuate this difference, free surface dissipating pressure gradients on the contrary of bottom floor, which tends to conserve and reflect them. 


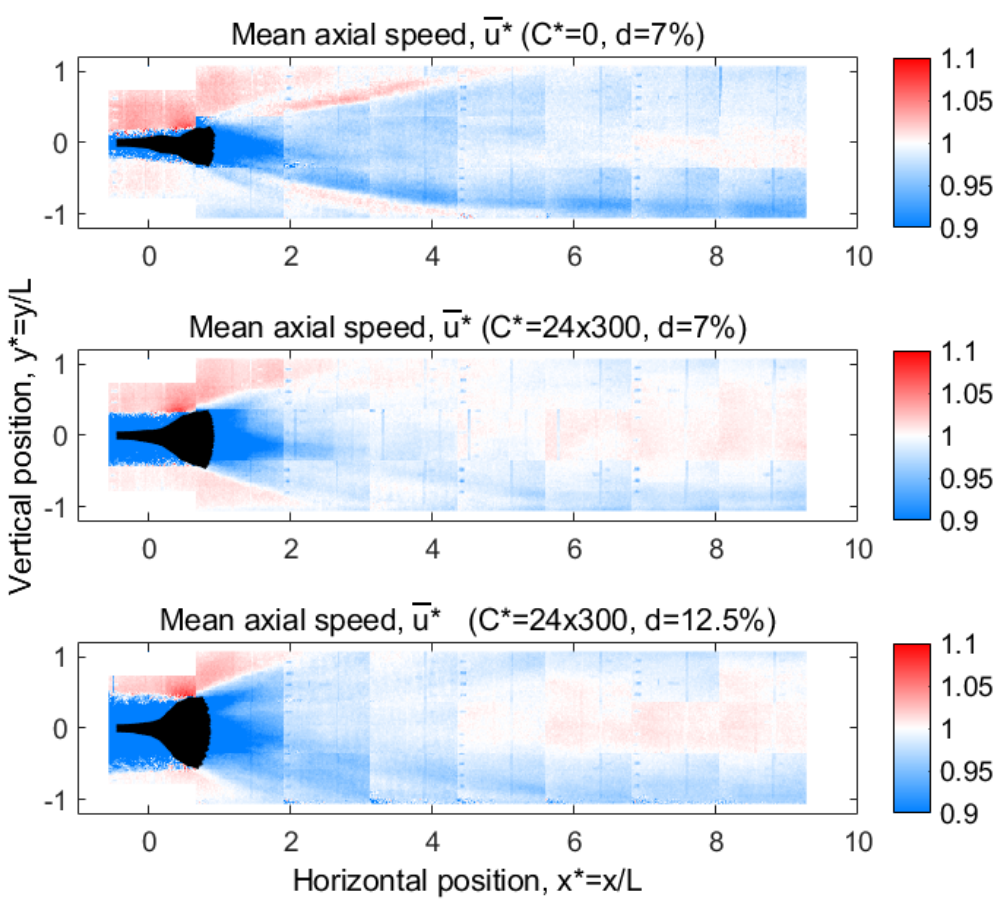

Figure 9: Mean axial velocity maps in the wake for the three tested configurations, C1 to C3 from top to bottom.

Mean velocity maps give essential information on the wake but, by definition, they do not take fluctuations into account. Therefore, Figure 11 shows current profiles of mean, maximum and minimum axial and vertical velocity in the wake of the third configuration (the most efficient). The minimal and maximal values presented here are calculated as being the first and the last percentile of the velocity signals. Indeed, this enables to neglect the outliers data points that would not have been removed by UOD method.

Figure 11 shows important velocity variations in the axial direction, of the order of $-0.3 \leq u^{*} \leq 0.3$ in the near wake. These variations fade away as they go further from the undulating membrane tidal energy converter. Fluctuations then maintain a level around $-0.1 \leq u^{*} \leq 0.1$, from $x=5 L$. Vertical fluctuations are even more important, of the order of $-0.5 \leq v^{*} \leq$ 0.5 in the near wake. They persist quite far in the flow, being still of the order of $-0.2 \leq v^{*} \leq 0.2$ at $x=8 L$. 

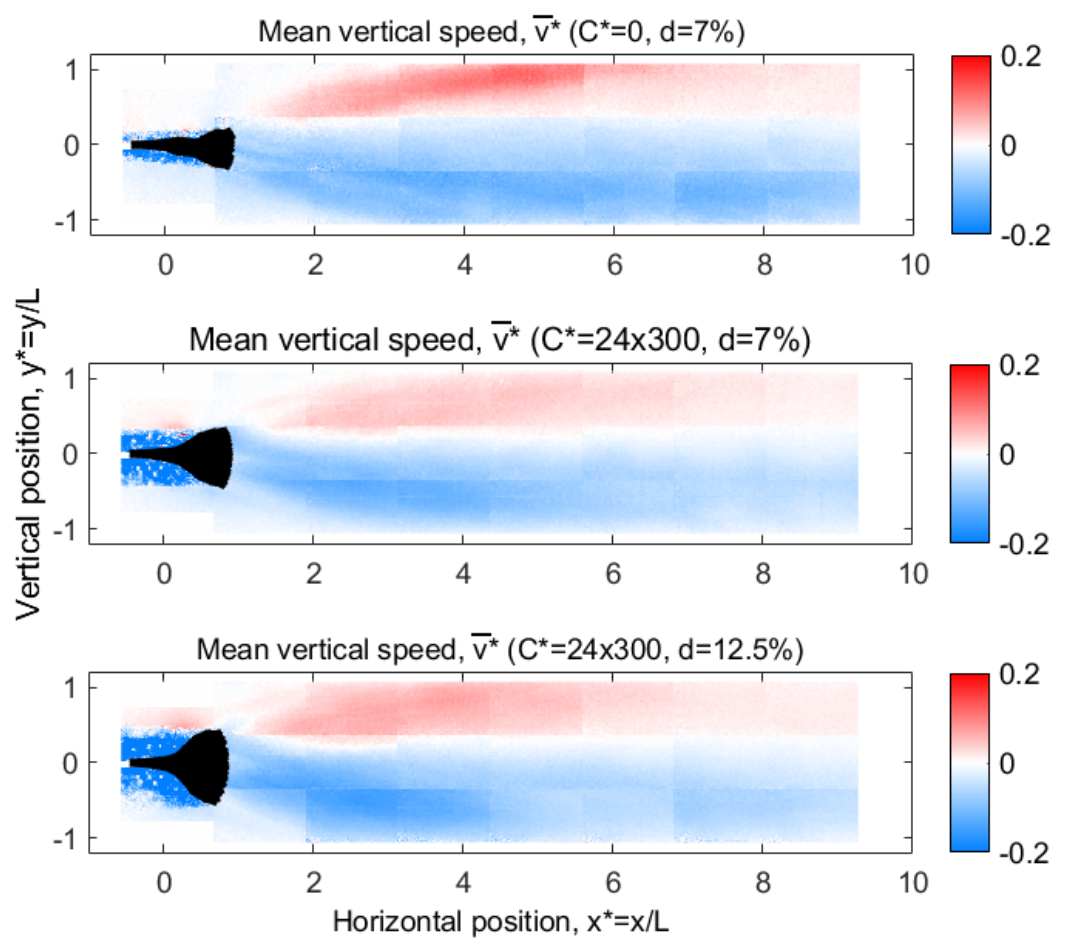

Figure 10: Mean vertical velocity maps in the wake for the three tested configurations, C1 to C3 from top to bottom.

Another way to quantify fluctuations levels in the flow is to calculate the turbulence intensity as shown in Figure 12. The gravity effect can be seen clearly for all configurations. Indeed, gravity leads to a faster downwards motion than upward, implying a thicker "bottom wake". The high turbulence intensity area $\left(I_{2 D} \geq 10 \%\right)$ fades away from $x=5 L$ after the trailing edge.

However, the area in the middle of the water column is much less impacted by the wake and low-level turbulence is there restored as soon as $x=3 L$ after the trailing edge. It could then be a favourable area for a downstream tidal converter, assuming side effects have little influence and that membrane interaction is undesirable. Indeed, there is few fluctuations and a little axial velocity deficit there. 

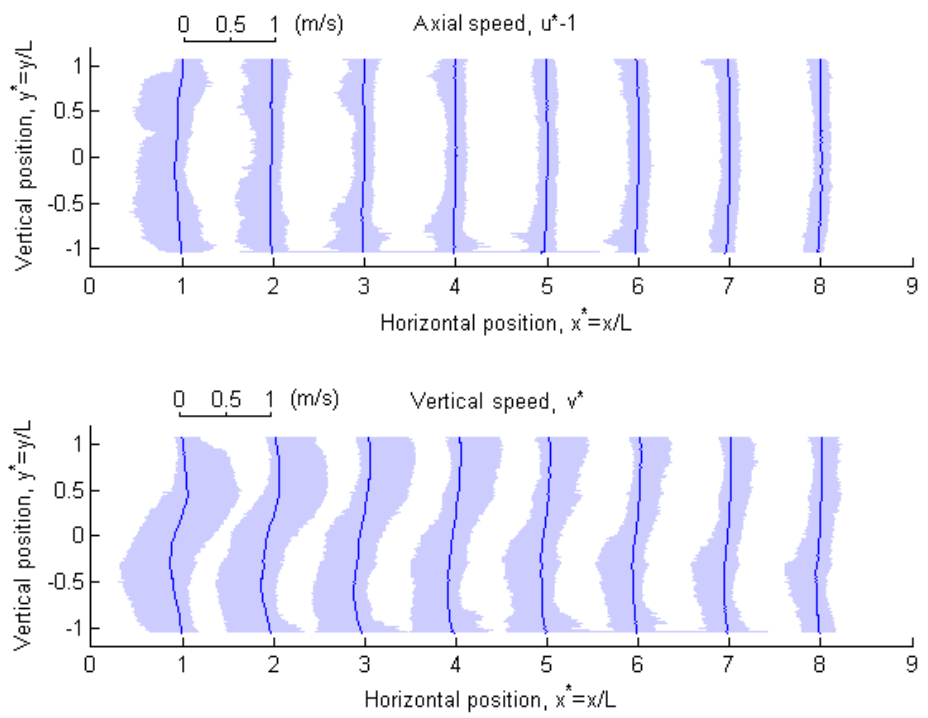

Figure 11: Axial (top) and vertical (bottom) mean velocity profiles (dark blue line) and associated fluctuations (light blue area) in the wake of the configuration C3.
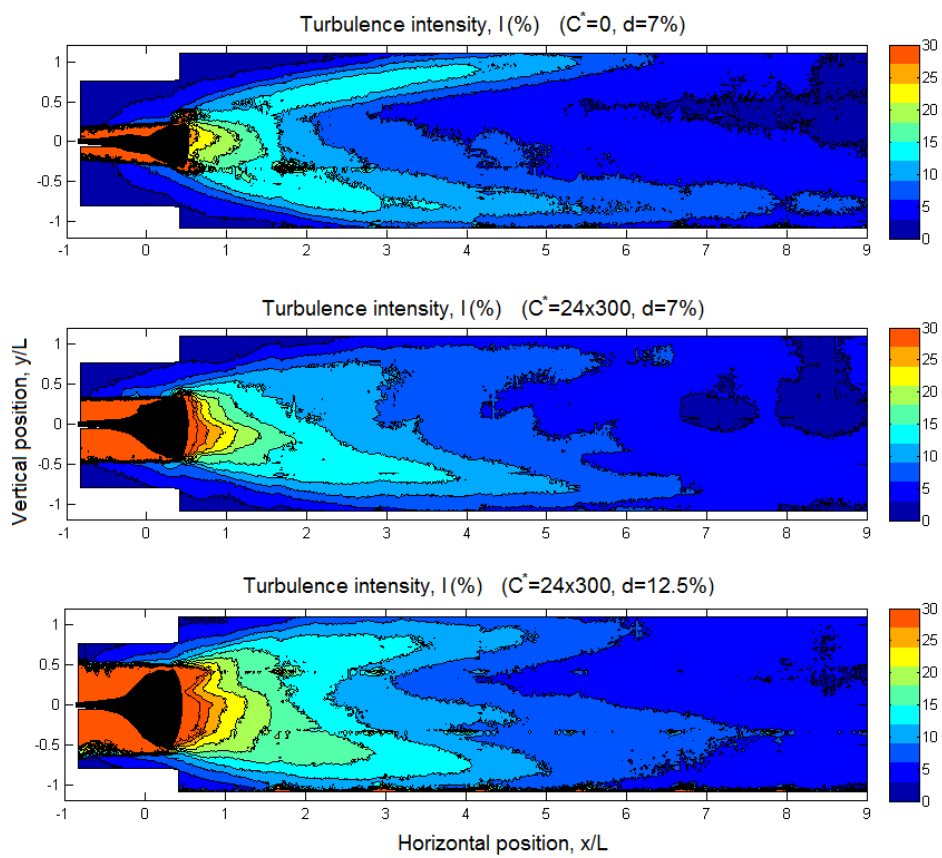

Figure 12: Mean turbulence intensity in the wake of the three tested configurations, C1 to C3 from top to bottom. 
In the objective to observe and understand more precisely the time evolution of the flow, it has been chosen to show the velocity signals in three points : A $(2 L, 0)$; $\mathrm{B}(4 L, 0)$ and $\mathrm{C}(4 L, 0.75 L)$. These points have been selected in order to be respectively in the near wake of the membrane, in a potential location for a second downstream tidal converter and in the way of an emitted perturbation. They are represented in Fig. 13, where an estimation of the perturbations trajectory is given by the parabola.

Velocity signals at these locations are presented in Figure 14 for the configuration C3. In the near wake of the membrane, in $\mathrm{A}(2 L, 0)$, the vertical velocity oscillates around $v^{*}=0$, at the undulating frequency of the membrane, between $-0.4 \leq v^{*} \leq 0.4$. It follows approximatively the vertical speed of the membrane's trailing edge (Fig. 15). Axial velocity in the same localization presents deficit peaks of around $40 \%$ twice a cycle (where $\mathrm{u}^{*} \approx$ 0.6 ), at the instant where the vertical velocity passes through $v^{*}=0$. Axial velocity is relatively little perturbed the rest of the time $\left(u \approx u_{\infty}\right)$.

Further in the wake, in $\mathrm{B}(4 L, 0)$, the axial velocity is almost not any more impacted. Vertical velocity evolves the same way as in $\mathrm{A}(2 L, 0)$, but its amplitude is reduced $\left(-0.2 \geq v^{*} \geq 0.2\right)$. Indeed, the main perturbations goes above and behind this point, and only the passage of the perturbations' rim is visible on this signal.

The third graph shows the velocity signals in $\mathrm{C}(4 L, 0.75 L)$, which is as the same downstream distance but on the trajectory of the perturbations. It is interesting to notice that vertical velocity in this point is equal to $v^{*}=0$ most of the time for each period, except when a perturbation passes through, where it rises temporally up to $v^{*}=0.4$. However it never becomes negative. The transit time of a perturbation is about $1 / 3$ of a period, being 1 second. By multiplying this transit time by $u_{\infty}$, the size of the perturbations in this point can be estimated to be of the order of the membrane's length. Axial velocity is more impacted in $\mathrm{C}(4 L, 0.75 L)$ than in $\mathrm{B}(4 L, 0)$ : it presents deficit peaks of about $\Delta u^{*}=-0.25$ during the perturbations transit. 


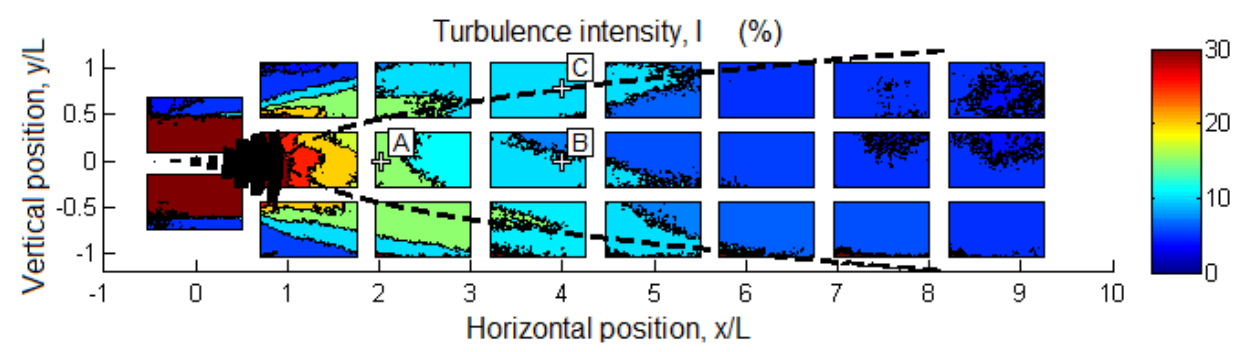

Figure 13: Position of the wake study points (A,B and C, located at the black cross) on the turbulence intensity map of third configuration. The dotted line parabol is an estimation of the perturbations trajectory.
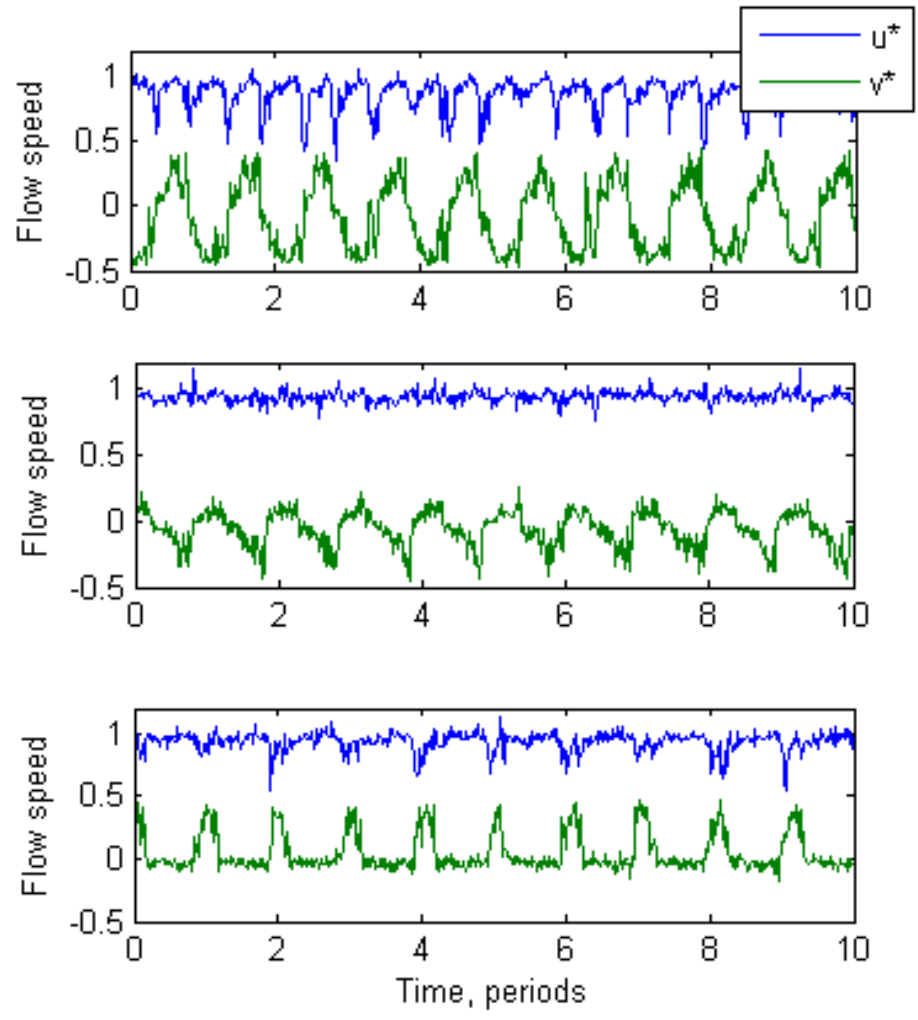

Figure 14: Axial and vertical velocity signals at the points $\mathrm{A}(2 L, 0) ; \mathrm{B}(4 L, 0)$ and $\mathrm{C}$ $(4 L, 0.75 L)$ from top to bottom for C3 


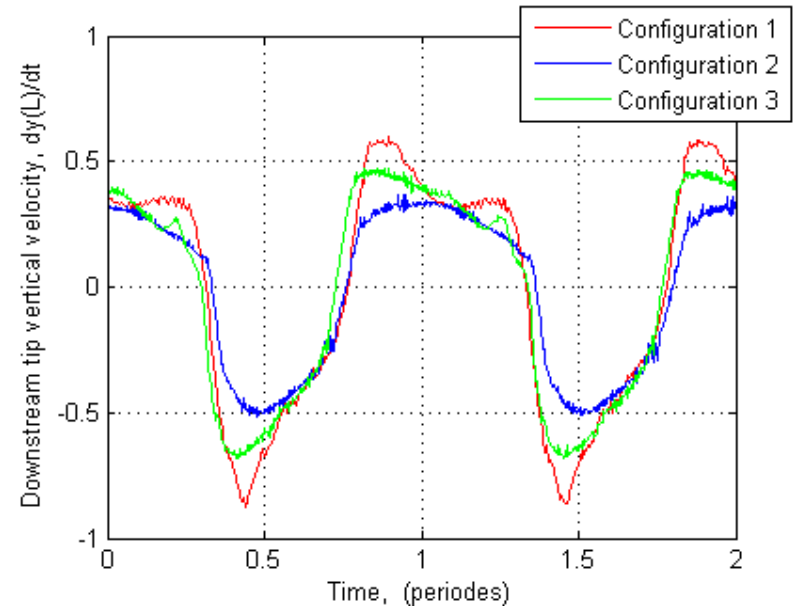

Figure 15: Results of downstream tip vertical velocity for the three tested configurations during two periods.

\subsection{Phase-averaged wake maps}

Phase-averaged maps of axial and vertical velocity as well as vorticy are presented for the configuration C3, respectively in Figures 16, 17 and 18. Ten periods are used to calculate the phase average, which is enough to limit the calculus' size while keeping a good precision. Spatial average of the standard deviation of local flow velocity from a period to another is less than $1 \%$.

Figure 16 represents phase-averaged axial velocity. A velocity deficit of sinusoidal shape is visible in the wake, with more important speed reduction in the top and the bottom of the tank. There is a strong deficit area in the near wake, which dissipates while going further from the membrane. This figure enables to better visualize the origin and propagation of velocity fluctuations observed in Figure 11.

In Figure 17 we can notice water packs projection of positive (resp. negative) vertical velocity once a cycle each in the top (bottom) part of the tank. These areas are about the size of the membrane and are moving downstream at mean upstream velocity speed $u_{\infty}$. The analysis of Figure 14 is then confirmed. They are submitted to confinement and interact with the free surface and the tank bottom. Indeed, bottom perturbations are more persistent, whereas upper vortices get to burst at the free surface. 
Vorticity maps presented in Figure 18 show a vortex ribbon shedding from the membrane's end and presenting a sinusoidal shape. It has noticeable similarities with undamped, unbuckled passive flag fluttering in axial flow [27]. Some differences come from the buckling cables that gives here a control over motion amplitude [23]. The strain they create in the membrane makes its curvature inversion require more strength. The membrane then slows down where it reaches the position corresponding to time $t=0$, and until enough pressure is generated by the flow. Afterwards, the membrane inverts its curvature and changes direction at $t=T / 6$, emitting a perturbation in the wake. These perturbations are certainly three-dimensional vortices for which only one component is visible in Figure 18. Vortex pairs have also been noticed. They should be further investigated as they can have their own self-induced behaviour [12].

Phase-averaged velocity maps for configurations $\mathrm{C} 1$ and $\mathrm{C} 2$ are not presented in this paper but phase-averaged vorticity maps calculated at the same moment of the cycle are presented in Figure 19 for every tested configurations. The wake is similar in the three cases but some differences are noticeable. The wake of the undamped configuration $\mathrm{C} 1$ is composed of smaller perturbations with higher vorticity, which persist further in the flow. These perturbations are emitted at a higher frequency because of the undulating frequency being higher. The wake of the "intermediate" configuration $\mathrm{C} 2$, for which the membrane is damped and has a smaller pre-strain, differs little from the one presented in Figures 16, 17 and 18.

Vortex ribbons match axial velocity deficit and go through positive and negative vertical velocity areas. The most intense vortices are located where the axial velocity deficit is the highest and where vertical velocity areas interact with free surface and tank bottom. In relation with Figure 1, the wake of an undulating membrane tidal energy converter is a combination between a passive flag's wake and a propulsive wake [14]. These results are consistent with vorticity maps obtained by numerical simulations in [6].

The vortex ribbon in the wake reminds the vortex model developed by [11], which express the flow as the sum of a constant current speed and perturbations modelled as point vortices that can be emitted in the wake. An example of this model for a passive flag is used in [18]. Our system is different mainly because of the heavier fluid, the pre-stress cables that enable a motion amplitude control and the dampers, which can change the membrane's frequency. Both parameters impact the wake development, as it is visible in the different mean and instantaneous wake maps. Such a vortex model for the undulating membrane tidal energy converter is presented in $[20]$. 

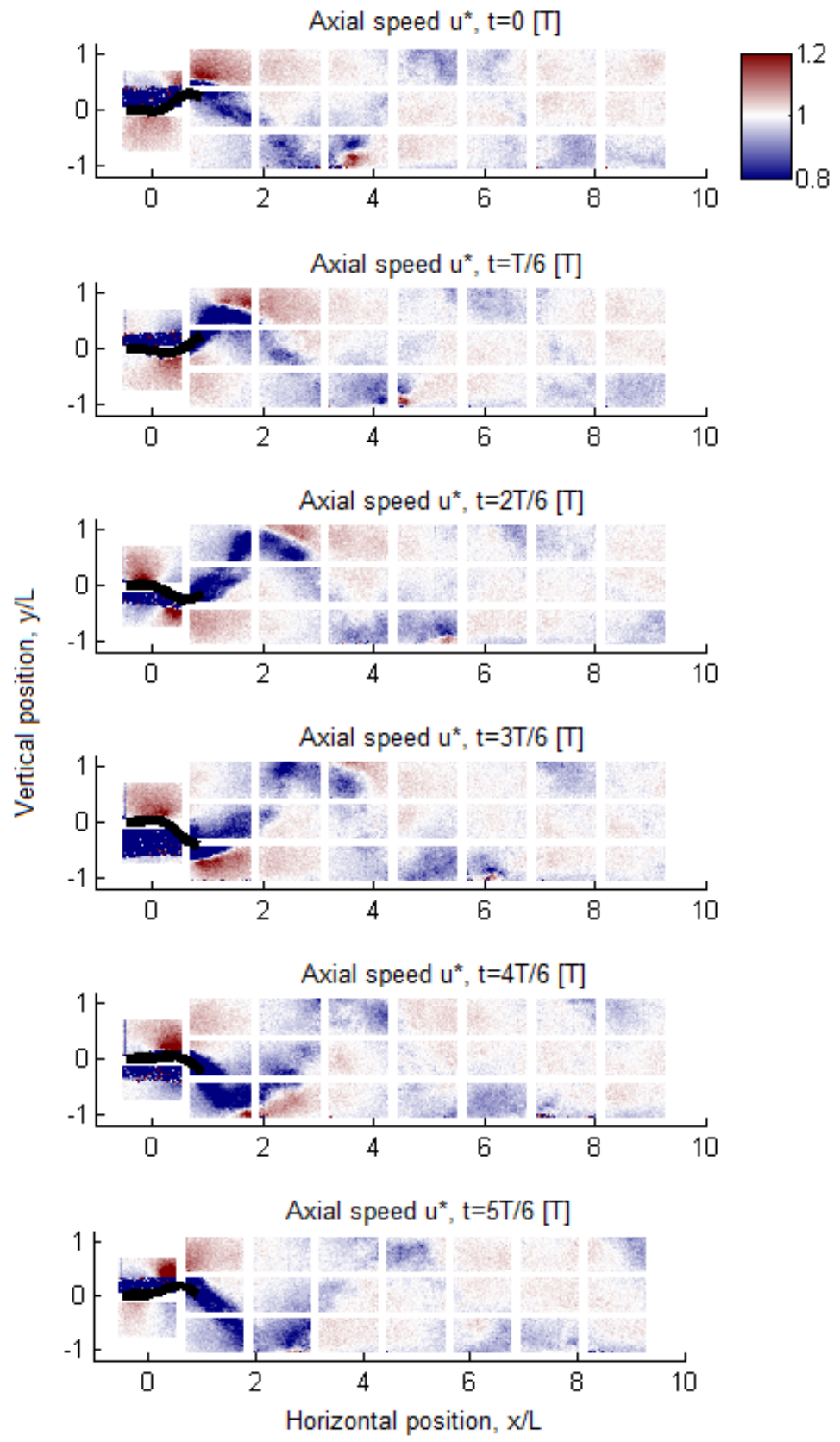

Figure 16: Phase-averaged axial velocity map for C3. 

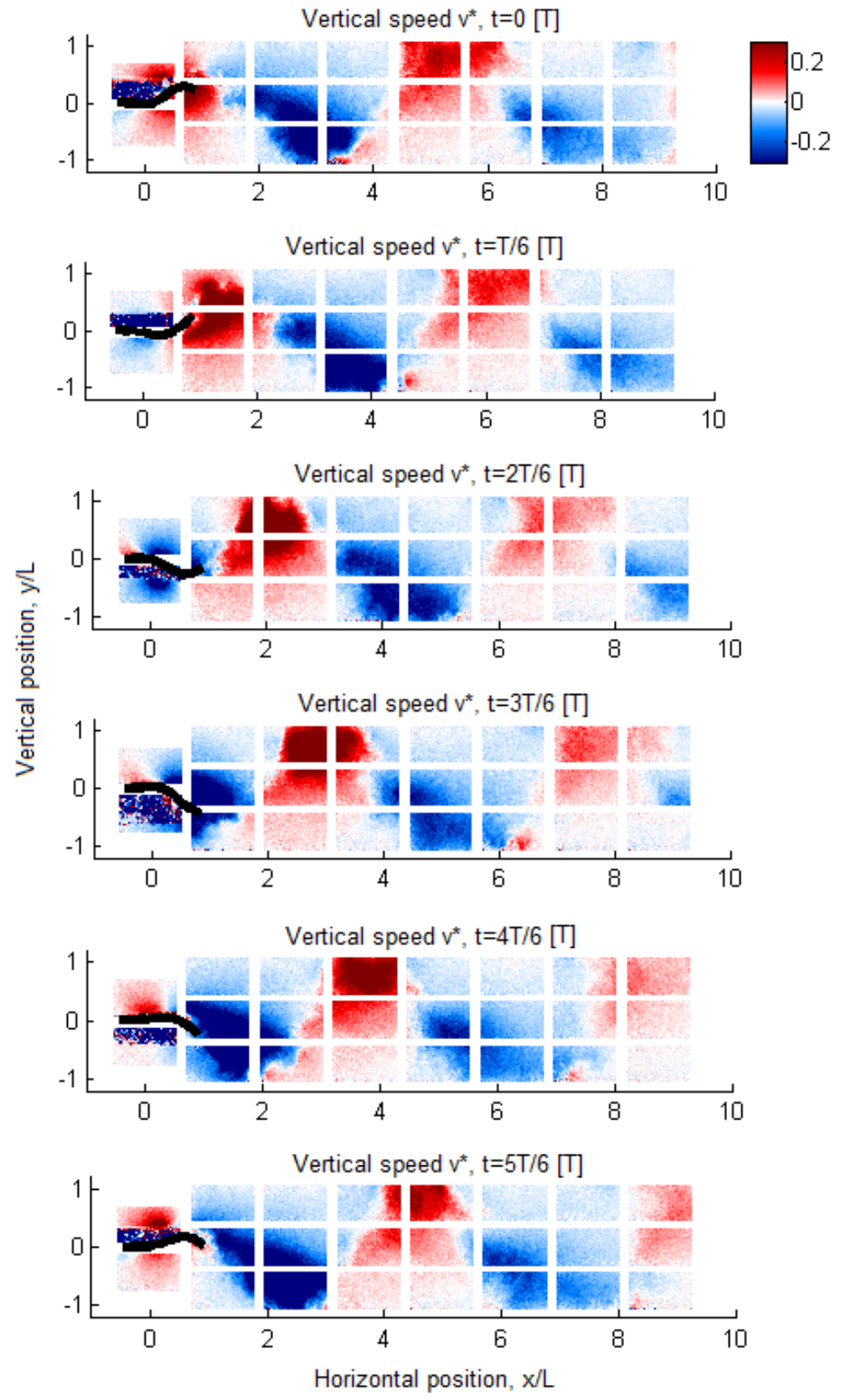

Figure 17: Phase-averaged vertical velocity map for C3. 

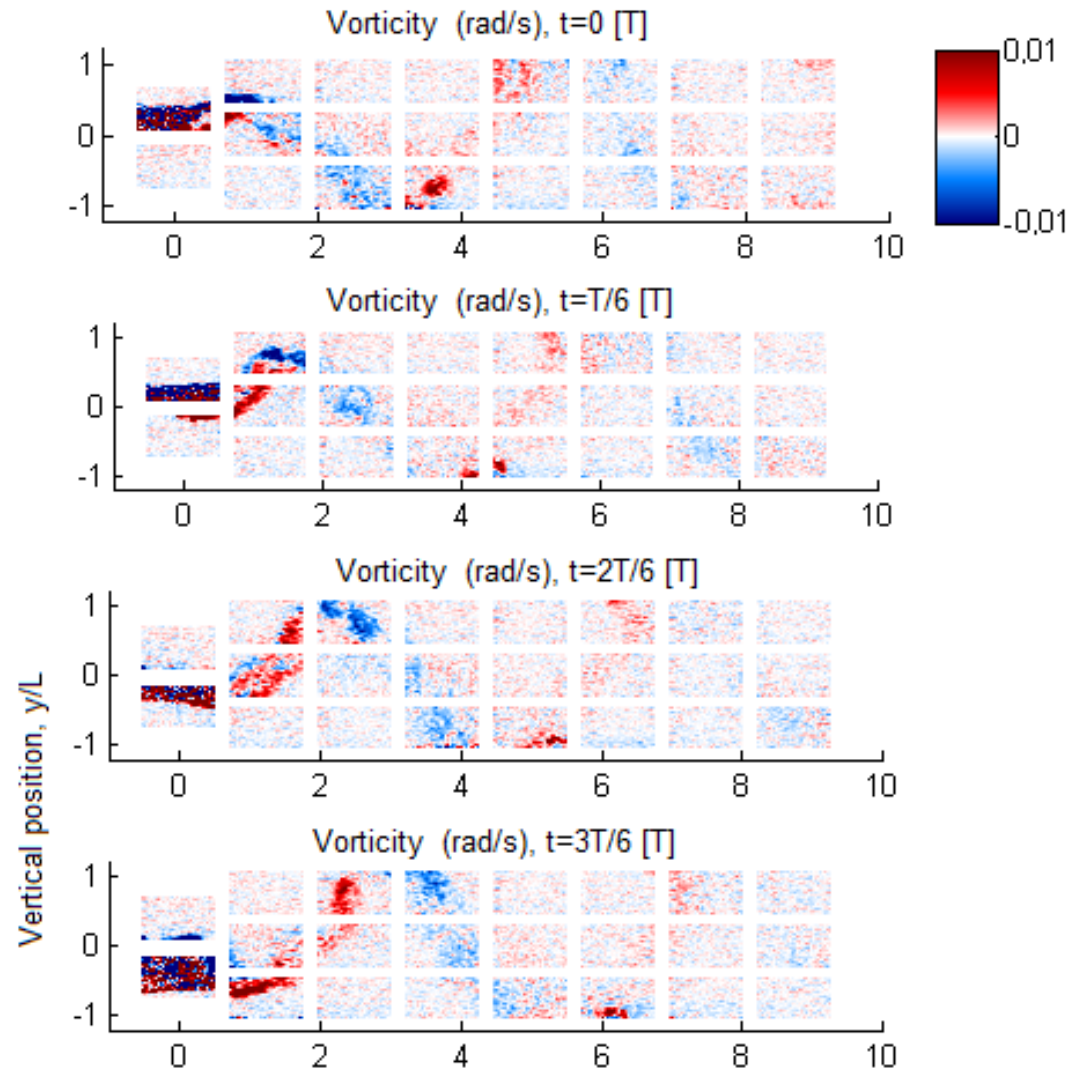

Vorticity $(\mathrm{rad} / \mathrm{s}), \mathrm{t}=4 \mathrm{~T} / 6[\mathrm{~T}]$

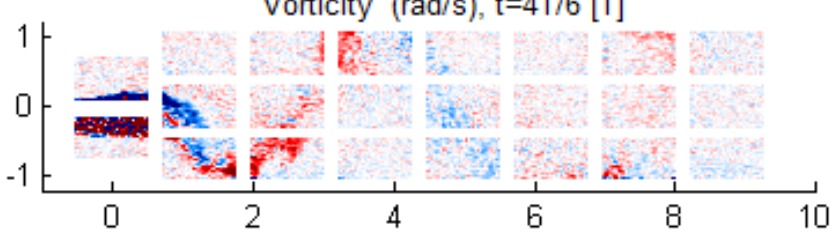

Vorticity $(\mathrm{rad} / \mathrm{s}), \mathrm{t}=5 \mathrm{~T} / 6[\mathrm{~T}]$

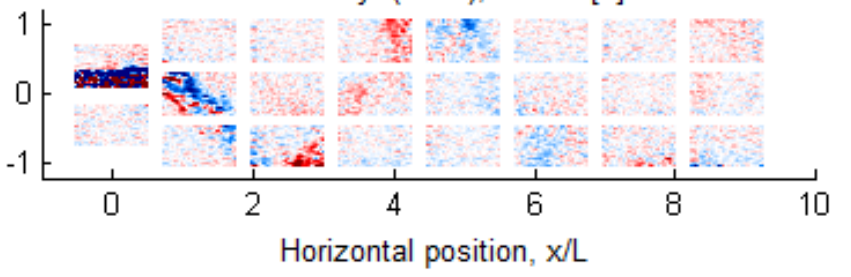

Figure 18: Phase-averaged vorticy map for C3. 

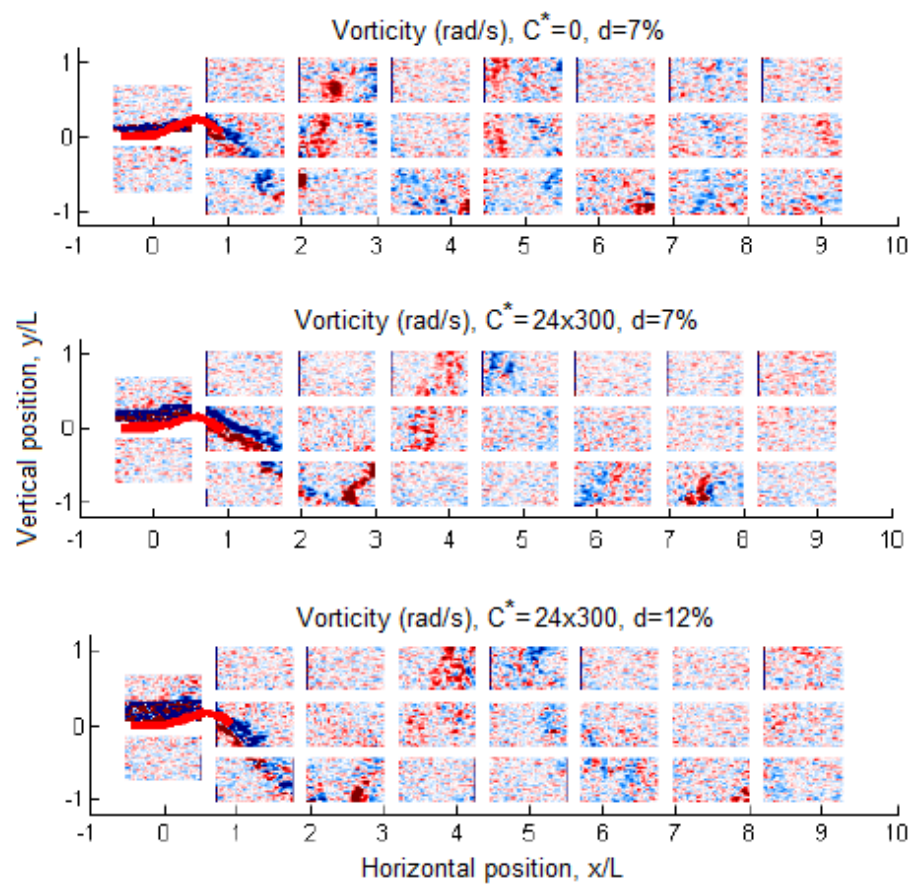

Figure 19: Phase-averaged vorticity maps comparison of tested configurations ( $\mathrm{rad} / \mathrm{s})$.

\subsection{Wake vortices characterization}

Figure 20 shows a vortex emitted by the membrane for every tested configurations. The selected vortices are centered in $x=2.6 \mathrm{~L}$ and emitted downward. Velocity vector fields and the angular velocity according to the distance to the center are presented for each vortex. A negative distance to the center means the point is placed at the left (upstream) of the center, whereas a positive distance indicates it is placed at the right (downstream) of the center. We can observe that the selected vortices are non-symmetrical so we may conclude that they are generated by the interaction between a negative velocity area and an area without vertical speed. They also have different profiles, the first configuration vortex being less intense but more organized, it is closer to a Lamb-Oseen profile than the two others (Eq. 7).

To give an example, an approximation with the least square method is done on a vortex in the wake of the $\mathrm{C} 1$ configuration (Fig. 21). Therefore, its circulation and its characteristic length are calculated : $\Gamma=0.27 \mathrm{~m}^{2} / \mathrm{s}$ and $r_{c}=0.0420 \mathrm{~m}[12]$. Only the data points in an horizontal line passing by the vortex center are taken into account here, but the same results can be obtained for all the orientations. 


$$
u_{\theta}=\frac{\Gamma}{2 \pi r}\left(1-\exp \left(-\frac{r^{2}}{r_{c}^{2}}\right)\right)
$$
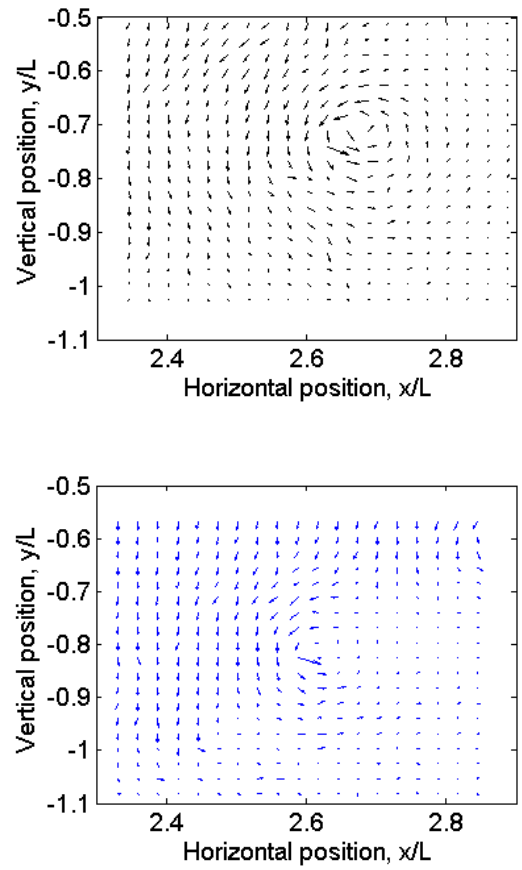

$\mathrm{C}$

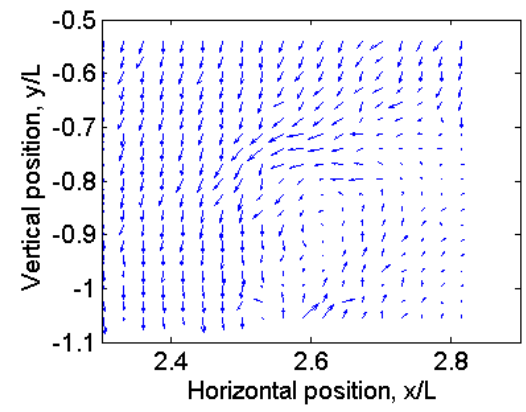

$\mathrm{b}$

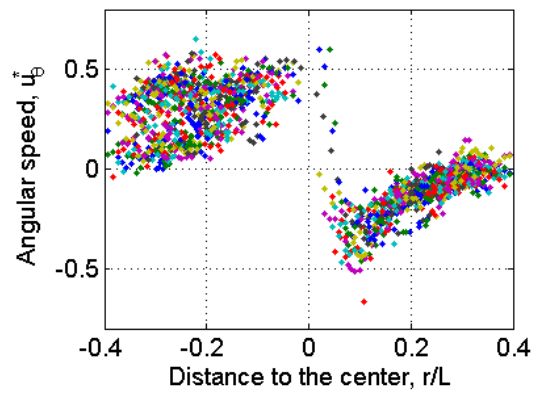

d
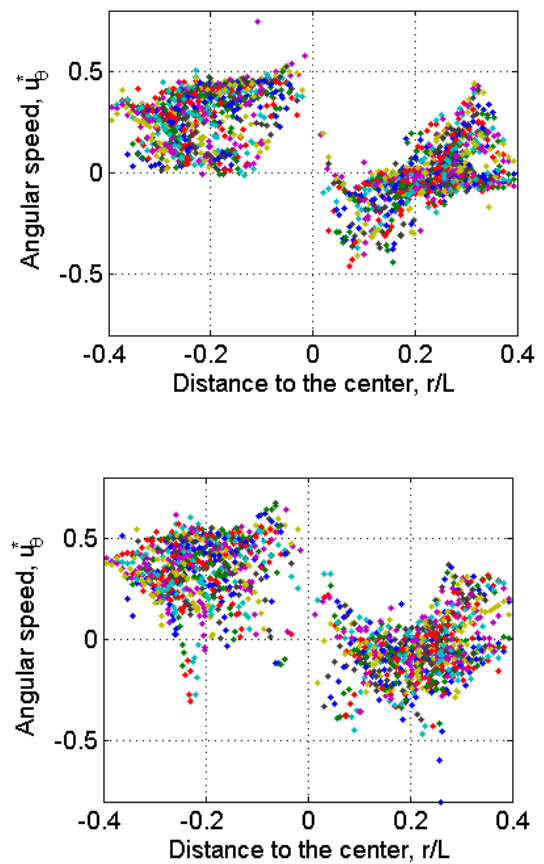

Figure 20: Examples of vortices emitted in the wake at $x=2.6 \mathrm{~L}$. Left : Vector field of pertubrated velocity $\left(u-U_{\infty}, v\right)$. Right : Tangential speed according to the distance to the vortex center. From top to bottom: C1 to C3. 


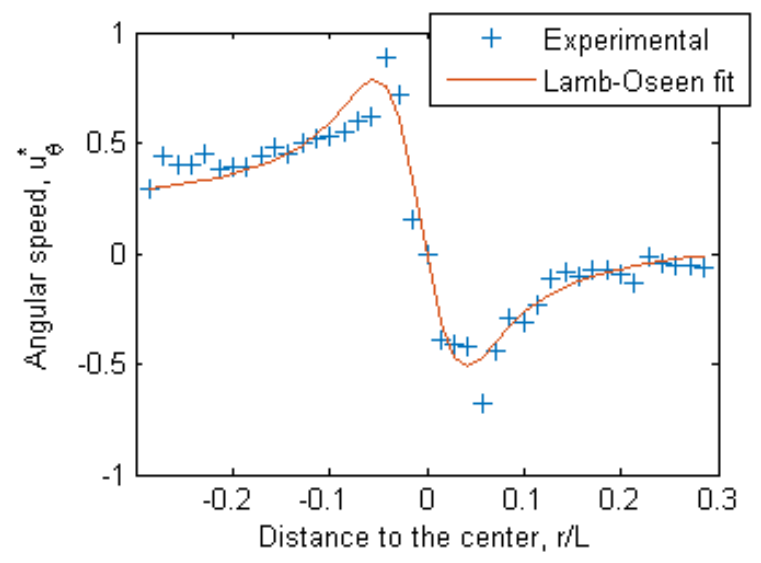

Figure 21: Angular speed on the horizontal line passing by the vortex center and its approximation with a Lamb-Oseen vortex formulation by the least square method.

It is impossible to follow the vortices position on several PIV planes that are not synchronized. A vortex that goes out from a plane is not the same that the one entering the following plane. So, it is difficult to have access to information such as their size, circulation or their time evolution [8], [9]. It is however possible to represent their trajectories on each plane. An example of the trajectory of vortex centres is represented in Figure 22 for the three tested configurations. The trajectories are regular and close to each others.

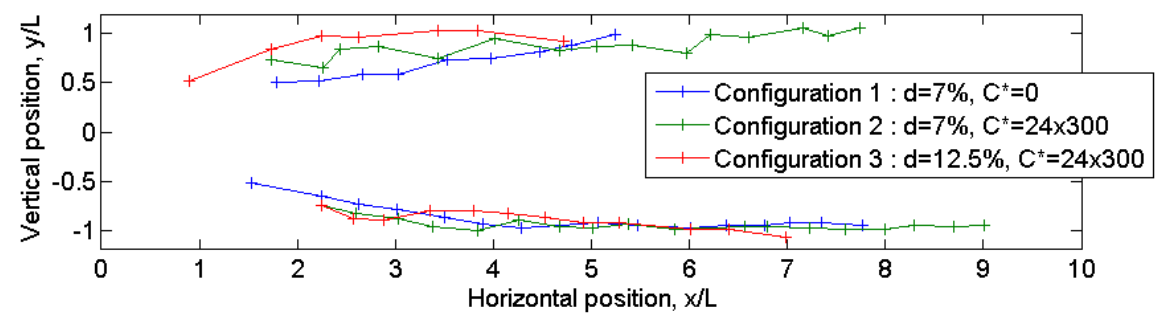

Figure 22: Example of trajectories of vortices emitted in the wake for the three tested configurations.

In order to estimate the dissipation of the perturbations in the wake, vertical maxima and vertical root-mean square of vorticity in the wake is plotted in Figure 23 according to horizontal position. An exponential decrease is noticed in both cases. This decrease is approximated with an equation of type : $\omega=\omega_{0} \exp \left(-k x^{*}\right)$, where $k$ is the dissipation rate, here equal to 0.125 . 
The velocity deficit and the turbulence intensity evolution in the wake of the membrane are presented in Figure 24 for three kind of evaluation. These results show that the mean axial velocity deficit is mostly restored after $x=5 L$ and the upstream flow is fully recovered after $x=8 L$.

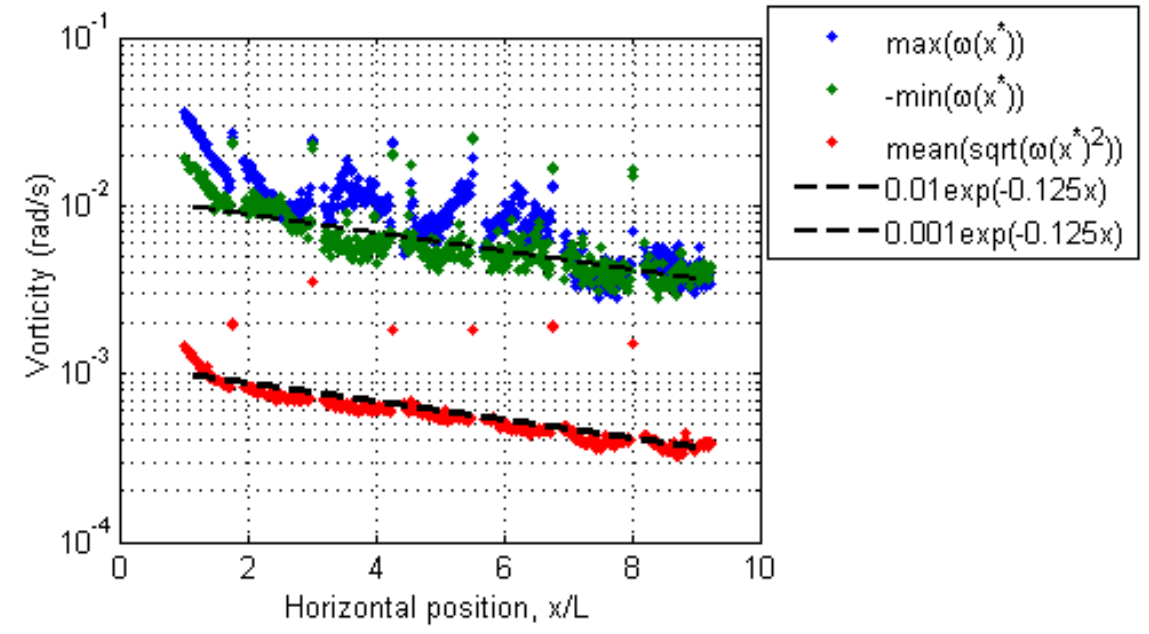

Figure 23: Vorticity dissipation in the wake of the second configuration
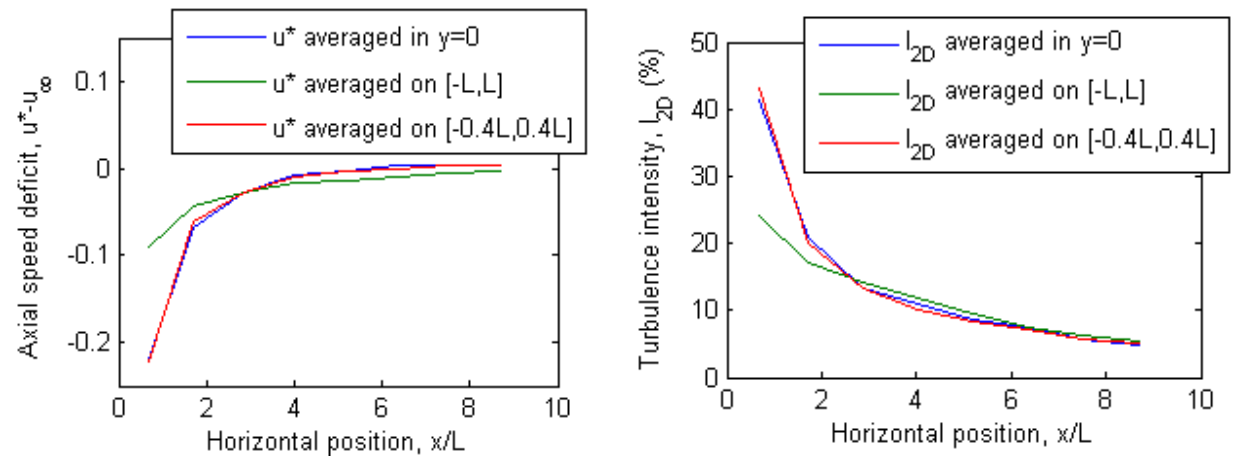

Figure 24: Velocity deficit and turbulence intensity in the wake averaged on $[-L, L]$, $[-0.4 L, 0.4 L]$ and at $y=0$ for C3 


\section{Conclusion}

PIV measurements have been performed in the wake of an undulating tidal energy converter. Three configurations have been tested: one undamped case (without PTO), one reference case and one with shorter cables and better performances. 23 planes have been acquired during $300 \mathrm{~s}$ for each configuration. A motion tracking system has been synchronized with the PIV measurements to phase the different planes in the wake of the membrane.

This wake characterisation gives information on the wake size, velocity deficit, turbulence and vorticity behind the membrane. Flow perturbations are emitted periodically and propelled vertically by the motion of the membrane's downstream part. The wake then interacts with free surface and tank bottom, which participates to its development and its dissipation.

The wake characterization synchronised with the membrane behaviour permits to identify at which moment of the undulation cycle the biggest perturbations are emitted. This instant is when the downstream tip reaches a vertical extremum, when the membrane slows down and changes its curvature direction. Perturbations are mainly composed of positive and negative vertical speed areas. An area of axial velocity deficit and positive/negative vorticity passes between them, as the vortex ribbon noticed by [27].

Three configurations have been tested in order to identify the influence of PTO and membrane's pre-stress on the wake's characteristics. This have led to the conclusion that the pre-stress drives the amplitude and the vertical speed of the membrane's undulation to be higher and to a more important vertical expansion of the wake. Furthermore, the damping induced by the power extraction slows down the membrane's motion, thus limiting the wake's length and intensity.

These measurements suggest that the best location for a downstream device to interact the less possible with an upstream device's wake would be at 5 to 8 membrane's length, in the same horizontal position. However, in open sea the spacing between two tidal converter will certainly be wider, due to industrial constraints during installation and maintenance. Furthermore, the turbulence intensity is more important in open sea, and this drives the flow speed in the wake to be recovered faster [7]. Therefore, this study suggests that the interaction effects for undulating membrane tidal energy converter are not predominant. 
Amplitude and interaction with tank limits impact wake vertical development. Indeed, the membrane vertical motion pushes flow perturbations upward and downward, but constrained boundaries limits the wake's vertical expansion. The rigid wall on the the floor forces the nearby flow to be horizontal, while free surface lesser perturbation intensity by dissipating pressure gradient. The effects of flow boundaries on the membrane have not been properly analysed here but the influence of free surface variations have been studied in [21], with the conclusion that they are not predominant. Furthermore, the tank depth of $2 \mathrm{~m}$ to test a $1 / 20^{\text {th }}$ scale prototype is representative of real-sea deployment conditions at full scale.

The effect of gravity is also visible in the wake. A way to evaluate the effect of both confinement and gravity would be to carry out the wake characterization with a vertically hanged membrane. However, such trials would be more complicated due to the lack of space to film the motion of the membrane with the motion tracking system and to characterize the flow from PIV measurements.

This study helps to prepare a tidal converter interaction study modelling a tidal farm. Results make possible to assume that the best downstream position for a second undulating converter should be at the same vertical position as the upstream one. Indeed, this area presents the less fluctuations in the flow. The perturbations being emitted up- and downward, they avoid this area, explaining its steadiness.

Results of this study are also useful to validate numerical models of the undulating tidal energy converter [20]. In particular, the dissipation rate can be used to calibrate the model, whereas wake maps, vortex circulation and velocity deficit will be compared with numerical results.

\section{Acknowledgment}

This work was supported by the French Environment and Energy Management Agency (ADEME), Eel Energy SAS and the MET-CERTIFIED project, receiving funding from the Interreg 2 Seas programme 2014-2020, co-funded by the European Regional Development Fund under subsidy contract $\mathrm{N}^{o} 2 \mathrm{~S} 01-020$.

\section{References}

[1] S. Alben. Wake-mediated synchronization and drafting in coupled flags. Journal of Fluid Mechanics, 641:489-496, 2009. 
[2] C. Carlier, B. Gaurier, G. Germain, G. Pinon, and E. Rivoalen. Three tidal turbines in interaction: an experimental study of turbulence intensity effects on wakes and turbine performances. under review, 2019.

[3] C. Carlier, G. Pinon, B. Gaurier, G. Germain, and E. Rivoalen. Numerical and experimental study of elementary interactions in marine current turbines array. In Proceedings of the 11th European Wave and Tidal Energy Conference, 2015.

[4] Y. Chen, B. Lin, J. Lin, and S. Wang. Experimental study of wake structure behind a horizontal axis tidal stream turbine. Applied energy, 196:82-96, 2017.

[5] A.H. Day, A. Babarit, A. Fontaine, Y-P. He, M. Kraskowski, M. Murai, I. Penesis, F. Salvatore, and H-K. Shin. Hydrodynamic modelling of marine renewable energy devices: A state of the art review. Ocean Engineering, 108:46-69, 2015.

[6] A. Déporte. Caractérisation du fonctionnement d'une hydrolienne á membrane ondulante pour la récuperation de l'énergie des courants marins. PhD thesis, Université de Bretagne Occidentale, 2015.

[7] B. Gaurier, C. Carlier, G. Germain, G. Pinon, and E. Rivoalen. Three tidal turbines in interaction: An experimental study of turbulence intensity effects on wakes and turbine performance. Renewable Energy, 148:1150-1164, 2020.

[8] L. Graftieaux, M. Michard, and N. Grosjean. Combining piv, pod and vortex identification algorithms for the study of unsteady turbulent swirling flows. Meas. Sci. Technol., 12:1422-1429, 2001.

[9] M. Ikhennicheu, G. Germain, P. Druault, and B. Gaurier. Experimental study of coherent flow structures past a wall-mounted square cylinder. Ocean Engineering, 182:137-146, 2019.

[10] S. Michelin J. Mougel, O. Doaré. Synchronized flutter of two slender flags. Journal of Fluid Mechanics, 801:652-669, 2016.

[11] J. Katz and A. Plotkin. Low speed aerodynamics. Campbridge University Press, 2001.

[12] T. Leweke, S. Le Dizès, and C. H. K. Williamson. Dynamics and instabilities of vortex pairs. Annual Review of Fluid Mechanics, 48:1-35, 2016. 
[13] B. Mallat, G. Germain, B. Gaurier, P. Druault, and J-Y. Billard. Experimental study of the bubble sweep-down phenomenon on three bow designs. Ocean Engineering, 148:361-375, 2018.

[14] U. Müller. Fish'n flag. Nature, 302:1511-1512, 2003.

[15] P. Mycek, B. Gaurier, G. Germain, G. Pinon, and E. Rivoalen. Experimental study of the turbulence intensity effects on marine current turbines behaviour. part i: One single turbine. Renewable Energy, 66:729$746,2014$.

[16] P. Mycek, B. Gaurier, G. Germain, G. Pinon, and E. Rivoalen. Experimental study of the turbulence intensity effects on marine current turbines behaviour. part ii: Two turbines in interaction. Renewable Energy, 66:729-746, 2014.

[17] S.A. Rolland, M. Thatcher, R. Ellis, B. Gaurier, T.N. Croft, and M. Crossn. Performance assessment of a vertical axis turbine in a marine current flume tank and cfd modeling. International Journal of Marine Energy, 12:35-45, 2015.

[18] L. Tang and M. Païdoussis. The influence of the wake on the stability of cantilevered flexible plates in axial flow. Journal of Sound and Vibration, 310:512-526, 2009.

[19] J. Thiébot, P. Bailly du Bois, and S. Guillou. Numerical modeling of the effect of tidal stream turbines on the hydrodynamics and the sediment transport : Application to the alderney race (raz blanchard), france. Renewable Energy, 75:356-365, 2015.

[20] M. Träsch, A. Chambon, A. Déporte, D. Lemosse, G. Pinon, E. Rivoalen, G. Germain, and J.-B. Drevet. Modelization of an undulating tidal energy converter. In Proceedings of the 13th European Wave and Tidal Energy Conversion, 2019.

[21] M. Träsch, S. Delacroix, A. Déporte, J.-B. Drevet, B. Gaurier, and G. Germain. Dynamic behavior of an undulating membrane tidal energy converter under wave and current loading. Ocean Engineering, page in press, 2020.

[22] M. Träsch, A. Déporte, S. Delacroix, G. Germain, B. Gaurier, and J.B. Drevet. Power estimates of an undulating membrane tidal energy converter. Ocean Engineering, 148:115-124, 2018. 
[23] M. Träsch, A. Déporte, J.-B. Drevet, and G. Germain. Impact of cables withdraw length on the dynamics of an undulating membrane tidal energy converter. In Proceedings of the 12th European Wave and Tidal Energy Conversion, 2017.

[24] E. Uddin, W.-X. Huang, and H.J. Sung. Interaction modes of multiple flexible flags in a uniform flow. Journal of Fluid Mechanics, 729:563-583, 2013.

[25] A. Vinod and A. Banerjee. Performance and near-wake characterization of a tidal current turbine in elevated levels of free stream turbulence. Applied energy, 254:113639, 2019.

[26] J. Westerweel and F. Scarano. Universal outlier detection for piv data. Experiments in Fluids, 39:1096-1100, 2005.

[27] J. Zhang, S. Childress, A. Libchaber, and M. Shelley. Flexible filaments in a flowing soap film as a model for one-dimensional flags in a twodimensional wind. Nature, 408:835-839, 2000. 